ganismen ${ }^{3}$ ). Ihr Studium wird vielleicht dazu beitragen, dab die einseitig mechanische Auffassung dieser wichtigen Erscheinungen, welche ihnen nur zum kleinsten Teile gerecht wird und deshalb zu halben und schiefen Anschauungen und Hypothesen fühtt, einer umfassenderen weicht, welche auch der vitalistischen Realität alles Seienden Rechnung trăgt. Für

3) 1907, Bd. XXIV, Heft 4; 1908, Bd. XXVI, Heft 1. die Kolloidchemie ist dieses aber von besonderer Bedeutung, da sit doch vorzugsweise mit Stoffen arbeitet, welche sie sich aus dem Gebiet des Lebendigen herausholt. Sic muB daher die tatsächlichen Beziehungen zum Lebendigen beherrschen und stets vor Augen haben, wovon bisher noch wenig zu merken ist.")

*) - falls man als einzig mogliche Bezichungen zwischen Kolloidchemie und Biologie die rom Verf. vertretenen Anschaunngen ansieht. Anm. A. Red.

\title{
Die Prioritätsansprüche des Herrn Max Münden.')
}

\author{
Von P. P. von Weimarn, St. Petersburg.
}

Dr. Max Münden schreibt selbst, dat er meine Ansicht, "daß der kristallinische $\mathrm{Zu}$ stand der einzige innere Zustand der Materie ist“" ), als sehr richtig anerkennt und bestreitet nicht, daß dieser Satz zum ersten Mal von

1) Koll,-Zeitschr. 3, 166 (1908).

9) Eine kurze his!orische Uebersicht uber die Entwickelung dieser Idee ist am Ende des ersten Kapitels des zweiten Teils meiner Abhandlung: "Zur Lehre von den $Z$ uständen det Materie" angefuthrt worden. Der Abdruck dieses Teils beginat in diesem Hefte. (Vgl auch meine kurzen Abhandlungen in der Koll.-Zeitschr. 2, 81, 218 (1907-1908). mir aufgestellt worden ist. Ich begreife daher nicht, auf welcheldee sich seine Prioritatsansprìche boziehen. Was die Prage der ldentităt der belebten und unbelebten Substanz anbetrifft, so habe ich mich nie speziell.mit ihr beschafftigt und bin weit davon enifernt, ein derartiges spezielles Problem losen $z u$ wollen.

St. Petershurg, 15. Dezember 1908.

Zur ev. sofortigen Rückäuferung ist die $M u ̈ n$. den'sche Einsendung Herrn Prof. Dr. v. Weimarn vorgelegt worden.

De Redaktion.

\section{Kolloidchemische Notizen.}

\section{Von Raph. Ed. L.lesegang.}

Ueber den Diamanten. Threlfall, welcher Graphit durch hohen Druck (100 tons) und Erhitzung auf die Schmelztemperatur der Magnesia nicht in Diamant verwandeln konnte, schliebt aus diesem Miberfolg; "Diamanten bedürfen zu ihrer Entstehuny bestimmter Kristallisationserreger, die man vielleicht durch Studium der Diamantasche finden kann." (Proc. chem. soc. 24, 5, 131, 1908.) … Ist es nicht wahrscheinlicher, dab man die Wirkung irgendwelcher Kolloidatoren ausschalten mut, um Kohlenstoff zur Kristallisation zu bringen?
In der Metallurgie ist andererseits die Gegenwart von Kolloidatoren vielfach erwünscht, weil sie das Kristallinischwerden der Metalle verhindern konnen.

Das Brücligwerden von beschwerter Seide usw., welches zum Teil auf das Auskristallisieren der zugegebenen Salze zurückgeführt wird, kann verzögert werden durch $\mathrm{Zu}$ gabe von geringen Mengen von hydrolytisch gespaltener Gelatine. (Die Gelatosen gehören zu den besten der bekannten Kolloidatoren.)

Referate.

\section{Arbeiten allgemeinen Inhalts.}

Galeotti, G., Ueber die sogenannten Metallverbindungen der Eiweißkorper nach der Theorie der chemischen Gleichgewichte. (Hoppe-Seylers Zeitschr. f. physiol. Chemie $\mathbf{4 0}$, $492,1904 . j$
Galeotti schliebt an die zuhlreichen Untersuchungen über Metallalbuminate an, die ergeben hatten, daß diese kaum als echte chemische Verbindungen aufgefaßt werden können. Durch eine Reihe von Untersuchungen über die ver- 